\title{
Emerging Two-Dimensional Crystallization of Cucurbit[8]uril Complexes: From Supramolecular Polymers to Nanofibers
}

\author{
Jesús del Barrio, ${ }^{*},, \diamond$ Ji Liu, ${ }^{\#}$ Ryan A. Brady, ${ }^{\ddagger}$ Cindy S. Y. Tan, ${ }^{\#, \otimes}$ Stefano Chiodini, ${ }^{\dagger \oplus ~ M a r i a ~ R i c c i, ~}$
} Rafael Fernández-Leiro, ${ }^{\perp}$ Ching-Ju Tsai, ${ }^{+}$Panagiota Vasileiadi, ${ }^{\mathbb{T}}$ Lorenzo Di Michele, ${ }^{\ddagger}{ }^{\ddagger}$ Didier Lairez, ${ }^{\S,}$ Chris Toprakcioglu, ${ }^{\text {,I }}$ and Oren A. Scherman* ${ }^{*} \odot$

${ }^{\dagger}$ Instituto de Nanociencia de Aragón, Universidad de Zaragoza, 50018 Zaragoza, Spain

$\diamond$ Departamento de Química Orgánica, Facultad de Ciencias, Instituto de Ciencia de Materiales de Aragón, CSIC-Universidad de Zaragoza, 50018 Zaragoza, Spain

${ }^{\#}$ Melville Laboratory for Polymer Synthesis, Department of Chemistry, University of Cambridge, Cambridge CB2 1EW, United Kingdom

${ }^{\ddagger}$ Cavendish Laboratory, Department of Physics, University of Cambridge, JJ Thomson Avenue, Cambridge CB3 0HE, United Kingdom

${ }^{\otimes}$ Faculty of Applied Sciences, Universiti Teknologi MARA, 94300 Kota Samarahan, Sarawak, Malaysia

${ }^{\perp}$ Spanish National Cancer Research Centre (CNIO), Melchor Fernández Almagro 3, 28029 Madrid, Spain

${ }^{+}$Laboratory of Biomolecular Research, Division of Biology and Chemistry, Paul Scherrer Institute, 5232 Villigen PSI, Switzerland

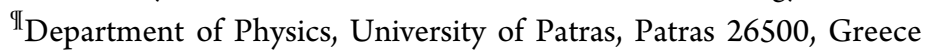

${ }^{\S}$ Laboratoire Léon Brillouin, CNRS, CEA, Université Paris-Saclay, 91191 Gif-sur-Yvette cedex, France

Laboratoire des Solides Irradiés, Ecole polytechnique, CNRS, CEA, Université Paris-Saclay, 91128 Palaiseau cedex, France

Supporting Information

ABSTRACT: The binding of imidazolium salts to cucurbit[8] uril, $\mathrm{CB}[8]$, triggers a stepwise self-assembly process with semiflexible polymer chains and crystalline nanostructures as early- and late-stage species, respectively. In such a process, which involves the crystallization of the host-guest complexes, the guest plays a critical role in directing self-assembly toward desirable morphologies. These include platelet-like aggregates and two-dimensional (2D) fibers, which, moreover, exhibit viscoelastic and lyotropic properties. Our observations provide a deeper understanding of the self-assembly of $\mathrm{CB}[8]$ complexes, with fundamental implications in the design of functional 2D systems and crystalline materials.

$\mathrm{T}$ he solution self-assembly of (macro)molecular building blocks provides a convenient pathway to a wide variety of nanoscale structures of broad utility. ${ }^{1}$ Notable examples include the aggregation of surfactants and amphiphilic block copolymers into micelles of various morphologies, ${ }^{2}$ as well as the assembly of specific DNA sequences into well-defined nanostructures. ${ }^{3}$ The architecture and functionality of such assemblies depend on both the molecular design of their individual constituents and the method for nanoscale aggregation. Emergent molecular building blocks can unveil alternative routes for the creation of functional assemblies and strategies to navigate multifaceted equilibria. One such system is the inclusion complexes of imidazolium salts and cucurbit$[n]$ urils, $\mathrm{CB}[n]$ s (Chart 1$){ }^{4}$ Our group has reported the binding of 1-methyl-3-[(naphthalen-2-yl)methyl]-imidazolium
Chart 1. Structures of $\mathrm{CB}[8]$ and Imidazolium Guests 1-3
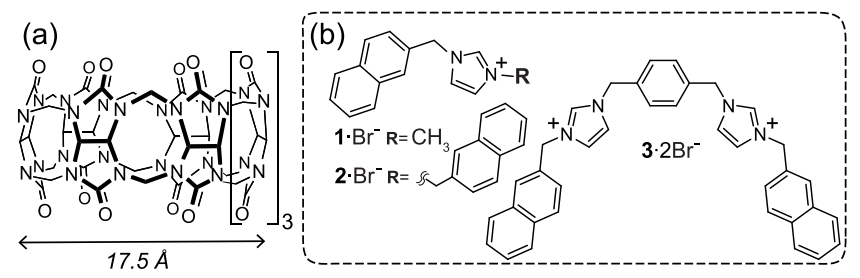

bromide ( $\mathbf{1}$ in Chart 1 ) to $\mathrm{CB}[8]$ to form a $\mathrm{CB}[8] \cdot \mathbf{1}_{2}$ homoternary complex. $^{5 a}$ More recently, other groups have reported the preparation of semiflexible $\mathrm{CB}[8]$-based supramolecular polymers by exploiting varied naphthalene-containing guest molecules. ${ }^{5 \mathrm{~b}}$

Herein, we report the complexation of naphthalene-derived imidazolium salts 2 and 3 (Chart 1 ) with $\mathrm{CB}[8]$. These specific host and guest molecules have the ability to engage in strong noncovalent interactions at the $\mathrm{mM}$ dilute concentrations yielding, unexpectedly, nanoscopic crystalline structures. These include platelet-like aggregates and two-dimensional (2D) nanofibers, which exhibit viscoelastic and lyotropic behavior.

Compound 2 features two equivalent $\mathrm{CB}[8]$-binding naphthalene residues bridged by one imidazolium group. The $\mathrm{CB}[8]$ complexation of 2 , similarly to that of $\mathbf{1}$, is quantitative in the $\mathrm{mM}$ concentration range. However, as shown by isothermal titration calorimetry (ITC), $\mathrm{CB}[8]$ and 2

Received: November 16, 2018

Published: August 19, 2019 
interact in a $1: 1$ ratio (Figure S1). Therefore, a theoretical equilibrium between bimolecular $1: 1$ and $n: n(n \geq 2)$ linear and cyclic species may be expected for an equimolar mixture of $\mathrm{CB}[8]$ and $2 .{ }^{6}$ The ${ }^{1} \mathrm{H}$ NMR spectrum of a 1:1 mixture of 2 and $\mathrm{CB}[8]$ contained a set of broad and ill-defined signals, which, as with many $\mathrm{CB}[8]$ complexes, were difficult to unambiguously assign to the presence of polymeric species.

In an attempt to clarify this characteristic, a series of morphological studies were performed on 1:1 mixtures of $\mathrm{CB}[8]$ and 2. The combined small-angle X-ray scattering (SAXS) and static light scattering patterns of an equimolar (1.0 $\mathrm{mM}$ ) mixture of $\mathrm{CB}[8]$ and 2 exhibits a clear power-law dependence of $q^{-2}$ in the high- $q$ region $(0.008 \AA<q<0.1 \AA)$, which is associated with the presence of semiflexible polymer chains of $\mathrm{CB}[8]_{n} \cdot \mathbf{2}_{n}$ (Figures S2 and S3). ${ }^{7}$ A radius of gyration $\left(R_{\mathrm{g}}\right)$ of ca. $30 \mathrm{~nm}$ was calculated by fitting the SAXS scattering intensity to the Debye function for Gaussian chains (Figures S2). Remarkably, the additional scattering at low $q$ values $(q<$ $0.005 \AA$ ) follows a $q^{-1}$ power law (Figures S3 and S4), which is a signature of rod-like structures. These results can be rationalized by assuming that the polymer chains of $\mathrm{CB}[8]_{n} \cdot 2_{n}$ coexist with some sort of larger rod-like structures. Indeed, elongated platelet-like aggregates were observed by atomic force microscopy (AFM images in Figures 1a, S9 and
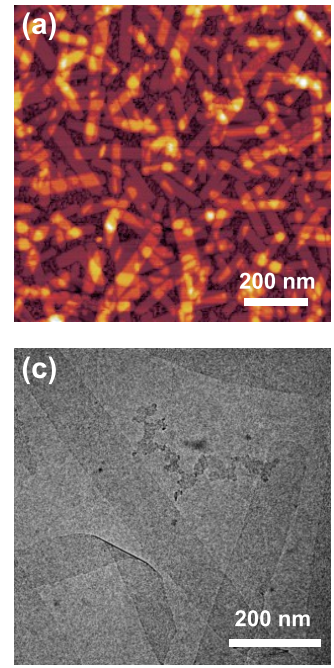
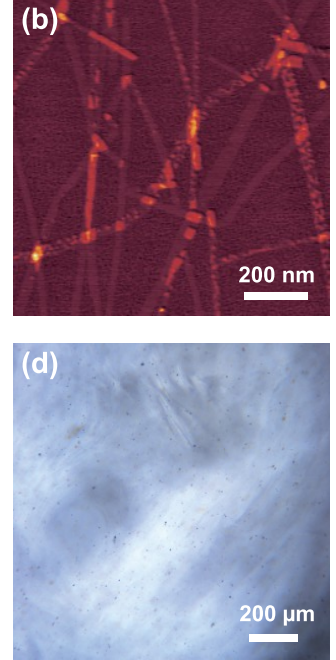

Figure 1. AFM morphological analysis of incubated samples of $\mathrm{CB}[8]+2$ (a) and $\mathrm{CB}[8]+3$ (b). Cryo-TEM micrograph of a sample of the $\mathrm{CB}[8]+3$ fibers $(\mathrm{c})$. Optical polarized micrograph of a $1.0 \mathrm{wt} \%$ aqueous solution of the $\mathrm{CB}[8]+3$ fibers $(d)$.

S12) and transmission electron microscopy (TEM images in Figure S22). Longitudinal height profiles showed that the aggregates exhibit a thickness between 2 and $4 \mathrm{~nm}$ (Figure S9).

We hypothesized that 3 , which is structural analogue of $\mathbf{2}$, could also produce polymers and higher-order assemblies in the presence of $\mathrm{CB}[8]$. A series of ITC experiments indicated that 3 and $\mathrm{CB}[8]$ preferentially interact in a $1: 1$ ratio (Figure $\mathrm{S} 1)$, similar to mixtures of 2 and $\mathrm{CB}[8]$. A combination of small-angle neutron scattering, AFM and TEM measurements showed the coexistence of small semiflexible polymer chains and large nanoscale assemblies in incubated samples of 3 and $\mathrm{CB}[8]$ (Figures $1 \mathrm{~b}, 1 \mathrm{c}$ and $\mathrm{S} 7$ ), echoing the behavior of mixtures of 2 and $\mathrm{CB}[8]$. $^{7}$ High aspect ratio fibers were clearly observed by AFM and TEM in aqueous equimolar (1.0 mM) mixtures of $\mathrm{CB}[8]$ and 3 (Figure $1 \mathrm{~b}, 1 \mathrm{c}, \mathrm{S} 10$ and S13).
The assembly of such small molecular weight building blocks into highly anisotropic micrometer-sized species is remarkable and prompted us to perform a series of control experiments aimed at shedding light on the fiber formation process. Samples obtained by casting aqueous solutions of $3(1.0 \mathrm{mM})$ alone onto mica substrates were completely free from fibers (Figure S14). Fibers were also absent from samples that were prepared immediately after mixing 3 and $\mathrm{CB}[8]$ (Figure S15). This suggested that both 3 and $\mathrm{CB}[8]$ are indispensable in the formation of fibers, a process which also has an associated incubation time (vide infra). Taking all the previous results into consideration, we propose that 1:1 mixtures of 3 and $\mathrm{CB}[8]$ transition through a two-step self-assembly process according to Figure 2a. Initially, the rapid host-guest

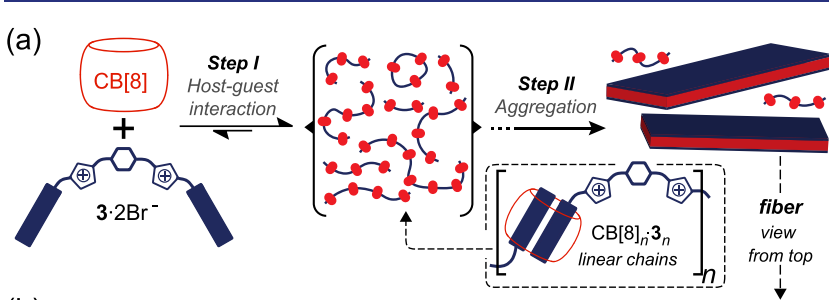

(b)

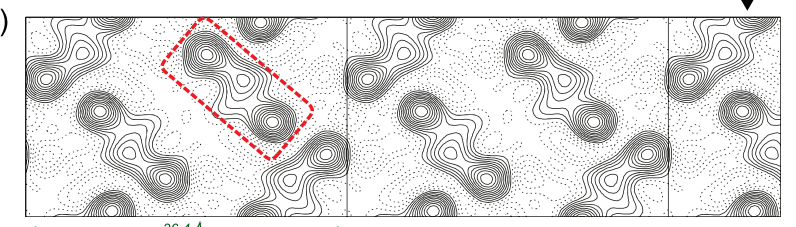

(c)

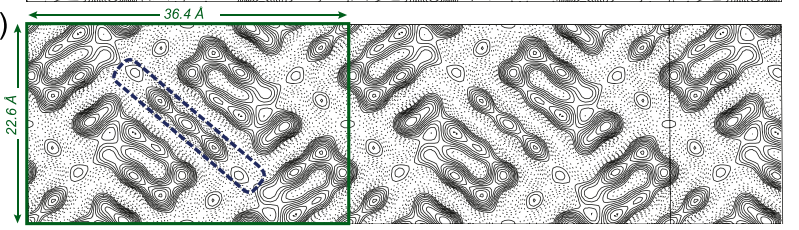

(d)

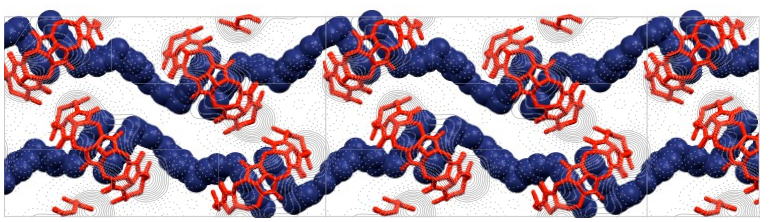

Figure 2. Step-wise self-assembly scheme (a) of $\mathrm{CB}[8]$ (in red) and 3 (in blue), cryo-TEM maps at a resolution of 5 (b) and $4 \AA$ (c), and packing model for the $\mathrm{CB}[8]+3$ fibers (d) superimposed to panel $b$. Unit cell parameters (in green) are shown in panel c.

recognition of 3 by $\mathrm{CB}[8]$ yields linear, and possibly also cyclic, polymer chains (Figure $2 \mathrm{a}$ ). At a later point, aggregation initiates which results in the formation of fibers. Such selfassembly phenomenon also applies to 1:1 mixtures of 2 and $\mathrm{CB}[8]$, which generate platelet-like aggregates instead of fibers.

It has recently been reported that the binding of specific guest molecules, such as viologen and cyanostilbene derivatives, to $\mathrm{CB}[8]$ results in the formation of various higher-order structures including three-dimensional organic frameworks and nanobundles. ${ }^{8 \mathrm{a}-\mathrm{e}}$ In our case, both 2 and 3, which share many similar structural characteristics (Chart 1 ), are able to produce large-scale aggregates in the presence of $\mathrm{CB}[8]$. We hypothesize that the aggregation is possibly driven by the combination of several factors including host-guest and hydrophobic interactions, as well as attractive interactions between the $\mathrm{CB}[8]$ molecules themselves. Highly elongated fibers grow in 1:1 mixtures of 3 and $\mathrm{CB}[8]$, whereas only relatively short platelet-like aggregates are obtained from those 
of 2 and $\mathrm{CB}$ [8]. Such divergent behavior, in analogy to other supramolecular systems, ${ }^{8}$ could be associated with the hydrophilic-hydrophobic ratio of the host-guest complexes (compound 3 features two positively charged imidazolium groups whereas 2 contains only one), ${ }^{9}$ and the packing of the host and the guest molecules within the aggregates.

With the idea of investigating such packing, we then imaged the fibers by means of liquid AFM and high-resolution (HR) cryo-TEM. The fibers have a length over several microns and a typical width of ca. $40 \mathrm{~nm}$. According to our liquid AFM analysis, the fiber thickness is $1.8 \mathrm{~nm}$ (Figures S10). Coulomb potential distribution maps obtained by HR cryo-TEM showed high density structural features which were attributed to the $\mathrm{CB}[8]$ molecules bound to the naphthalene residues of 3 (Figure 2b). Higher resolution maps showed additional features which were associated with the naphthalene-bridging linker of 3 (Figure 2c). A plausible packing model, considering the cryo-TEM maps and the fact that the outer diameter of $\mathrm{CB}[8]$ matches the thickness of the fibers, ${ }^{4 a}$ consists of a single-layer structure of host-guest complexes. In our model, individual $\mathrm{CB}[8]_{n} \cdot 3_{n}$ chains are laterally assembled into zigzagged strands, which run along the long fiber axis. The host molecules are organized in a "side to portal" arrangement (herringbone structure) with their equatorial planes orthogonal to the fiber main lattice. As such, adjacent $\mathrm{CB}[8] \mathrm{s}$ from individual strands are capable of engaging in multiple hydrogen-bonding interactions (between the carbonyl groups of the portals and the methylene and equatorial methine groups), ${ }^{10}$ a feature which likely contributes to stitching the $\mathrm{CB}[8]_{n} \cdot 3_{n}$ chains together (Figure S28). Ion-dipole and $\mathrm{C}-\mathrm{H} \cdots \mathrm{O}=\mathrm{C}$ hydrogen bonding interactions between the imidazolium groups of 3 and the carbonyl portals of neighboring $\mathrm{CB}[8] \mathrm{s}$ may also play an important role in reinforcing the lateral assembly of the strands.

Solution-phase X-ray diffraction (XRD) experiments of samples of the fibers revealed a series of relatively sharp peaks (Figure 3), which is characteristic of crystalline materials. ${ }^{11}$ The HR cryo-TEM data matches the XRD patterns and indicates that $\mathrm{CB}[8]_{n} \cdot 3_{n}$ crystallizes in the $P 22_{1} 2_{1}$ plane group symmetry (Figure S18 and Table S1). The platelet-like aggregates are also crystalline in nature

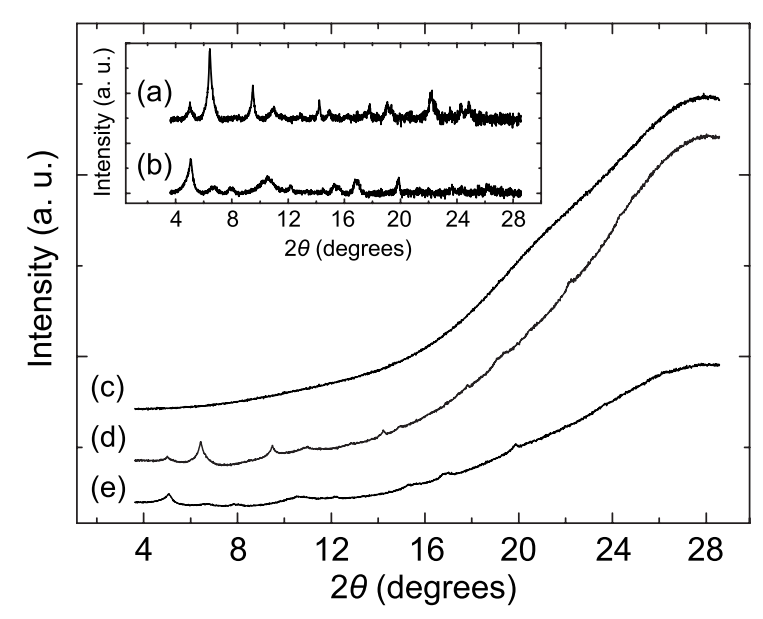

Figure 3. XRD patterns corresponding to aqueous solutions of the $\mathrm{CB}[8]+3$ fibers (a, d), the $\mathrm{CB}[8]+2$ platelet-like aggregates $(\mathrm{b}, \mathrm{e})$, and neat $\mathrm{H}_{2} \mathrm{O}(\mathrm{c})$. For clarity, data set in the inset $(\mathrm{a}, \mathrm{b})$ has been background-subtracted.
(Figure 3 and S22), and exhibit a different microstructure in comparison to the fibers. We have been, however, unable to suggest possible symmetry unit cells and parameters solely on the basis of our TEM and XRD results.

Samples of the fibers are free-flowing fluids but noticeably more viscous than samples of the platelet-like aggregates or 3 alone at analogous molar concentration. In a similar fashion to many suspensions of fibrillar aggregates, ${ }^{12}$ shear-thinning was observed in samples of the fibers (Figure S26). The linear viscoelasticity of an equimolar mixture of 3 and $\mathrm{CB}[8]$ (1.0 $\mathrm{mM}$ ) after incubation was characterized by monitoring the variation of the storage $\left(G^{\prime}\right)$ and loss moduli $\left(G^{\prime \prime}\right)$, at a constant strain, as a function of the angular frequency (Figure $4 a)$. At $20^{\circ} \mathrm{C}, G^{\prime}$ dominates over $G^{\prime \prime}$ above a critical frequency

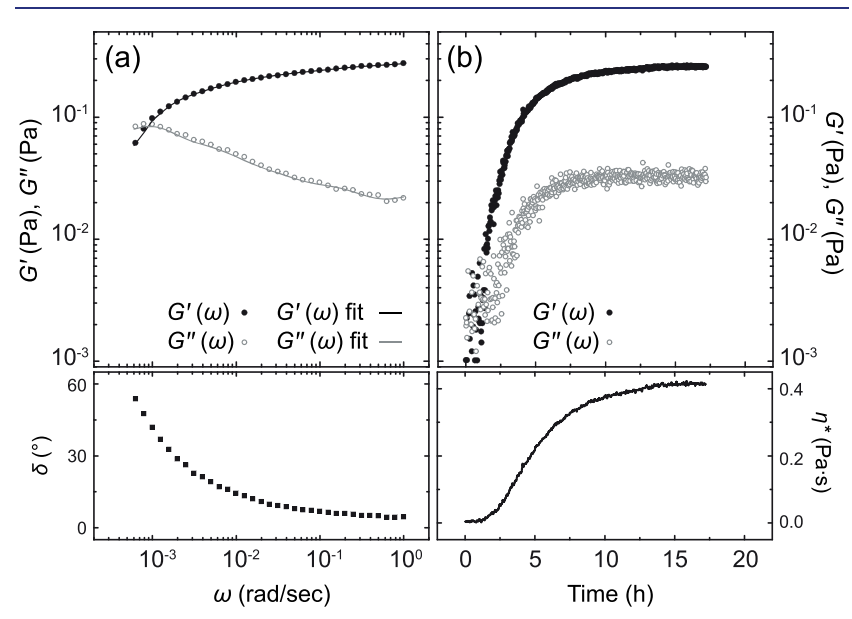

Figure 4. Variation of $G^{\prime}, G^{\prime \prime}$ and phase angle as a function of angular frequency, at $20^{\circ} \mathrm{C}$, for an equimolar $(1.0 \mathrm{mM})$ mixture of $\mathrm{CB}[8]$ and 3 after incubation, and fits (solid lines) to the Maxwell model with three elements (a). Variation of $G^{\prime}, G^{\prime \prime} \eta^{*}$ at $20{ }^{\circ} \mathrm{C}$ as a function of time for an equimolar $(1.0 \mathrm{mM})$ mixture of $\mathrm{CB}[8]$ and 3 (b). See Figure S25 and Table S2 for the fitting of the rheological data in (a).

value $\omega_{c}$; whereas $G^{\prime}$ crosses over and drops below $G^{\prime \prime}$ at frequency values below $\omega_{c}$. These trends are reminiscent of the Maxwellian behavior found in many viscoelastic worm-like micellar solutions, ${ }^{13}$ but differs from it at high angular frequency values $(\omega>0.002 \mathrm{rad} / \mathrm{s})$. When concentrated up to a dry matter content of ca. $1.0 \mathrm{wt} \%$, free-flowing samples of the fibers turned into gels, which furthermore exhibited lyotropic properties (Figure 1d). Such behavior is also distinctive of many fluids containing rod-like particles. ${ }^{14}$

The kinetics of fiber formation were also elucidated through a series of rheological measurements. We tracked the evolution of a freshly prepared equimolar mixture of 3 and $\mathrm{CB}[8]$ (1.0 $\mathrm{mM}$ ) by recording the complex viscosity, $\eta^{*}$, of the sample over time at a constant frequency and strain amplitude (Figure 4b). After an induction period, values of $\eta^{*}$ increase with time and then plateau (Figure $4 \mathrm{~b}$ ). As fibers were apparent from the morphological analysis, the initial $\eta^{*}$ increase is likely associated with the growth of the fibers, provided sufficient 3 and $\mathrm{CB}[8]$ are available. After the fibers reach a critical length, $\eta^{*}$ plateaus. The final values of $G^{\prime}, G^{\prime \prime}$ and $\eta^{*}$ are stable and match those of samples subjected to an incubation period of ca. $24 \mathrm{~h}$ at $20{ }^{\circ} \mathrm{C}$ (see Figure 4). The kinetic data corresponding to the initial increase of the viscoelastic moduli was analyzed according to the Avrami theory and a value of the Avrami exponent $(n)$ of approximately one was determined 
(Figure S27). ${ }^{15}$ Considering the existence of fibrillar aggregates, such a value for $n$ is consistent with a onedimensional and interfacial-controlled growth process. ${ }^{16}$

In summary, we have demonstrated how nanoscale assemblies of different morphologies and macroscopic properties can be prepared in a one-pot fashion from complementary components of subnanometer dimensions. Guest molecules 2 and 3 undergo a stepwise self-assembly process in the presence of $\mathrm{CB}[8]$, which yields anisotropic crystalline aggregates. Semiflexible polymer chains have been identified as intermediate species in the assembly mechanism. Micron-sized twodimensional fibers with a thickness of a single $\mathrm{CB}[8]$ macrocycle $(1.8 \mathrm{~nm})$ are produced from 1:1 mixtures of $\mathrm{CB}[8]$ and 3 , which is unprecedented in the field of $\mathrm{CB}[n]$-based molecular recognition. The molecular designs of our individual building blocks (pairs of host and guest molecules), encode structural information which translates to significant differences in aggregate morphology. This, in turn, has important implications in the macroscopic properties of the systems as illustrated by the identification of the viscoelastic and lyotropic properties of the fibers (Figures $1 \mathrm{~d}$ and 4).

These unique features, which arise from a combination of tight binding and crystallization-assisted self-assembly, will ensure a variety of applications. For example, supramolecular fibers made from low molecular weight components may become ideal materials as drag reducers or viscosity modifiers in applications where stimuli-responsive degradable systems are preferred over traditional high molecular weight polymers. ${ }^{17}$ Additionally, this fundamental study will enable the development of hierarchical crystalline nanostructures and functional $2 \mathrm{D}$ systems.

\section{ASSOCIATED CONTENT}

\section{S Supporting Information}

The Supporting Information is available free of charge on the ACS Publications website at DOI: 10.1021 /jacs.9b07506.

Experimental procedures, synthesis of guest molecules, preparation of complexes and additional characterization details (PDF)

\section{AUTHOR INFORMATION}

\section{Corresponding Authors}

*jdb529@unizar.es

*oas23@cam.ac.uk

\section{ORCID $\odot$}

Stefano Chiodini: 0000-0002-8842-9324

Lorenzo Di Michele: 0000-0002-1458-9747

Oren A. Scherman: 0000-0001-8032-7166

\section{Notes}

The authors declare no competing financial interest.

\section{ACKNOWLEDGMENTS}

This work was supported by the EPSRC (reference no. EP/ G060649/1), an ERC Starting Investigator Grant (project no. 240629, ASPiRe) and a Next Generation Fellowship from the Walters-Kundert Foundation. J.d.B. acknowledges the MINE$\mathrm{CO}$, the FSE and the FEDER for funding through projects RYC-2015-18471 (Ramón y Cajal program) and CTQ201784087-R. L.D.M. acknowledges support from a Royal Society University Research Fellowship UF160152. R.A.B. acknowl- edges support from the EPSRC CDT in Nanoscience and Nanotechnology (NanoDTC), grant number EP/L015978/1. The authors thank Dr. Sean Lovett for his help with the fitting of the oscillatory rheology frequency sweep data, Dr. Aurel Radulescu for his assistance with some of the SANS measurements and Dr. Silvia Hernández-Ainsa for helpful discussions.

\section{REFERENCES}

(1) (a) Whitesides, G. M.; Mathias, J. P.; Seto, C. T. Molecular SelfAssembly and Nanochemistry: a Chemical Strategy for the Synthesis of Nanostructures. Science 1991, 254, 1312-1319. (b) Aida, T.; Meijer, E. W.; Stupp, S. I. Functional Supramolecular Polymers. Science 2012, 335, 813-817.

(2) (a) Israelachvili, J. N.; Mitchell, D. J.; Ninham, B. W. Theory of Self-Assembly of Hydrocarbon Amphiphiles into Micelles and Bilayers. J. Chem. Soc., Faraday Trans. 2 1976, 72, 1525-1568. (b) Laughlin, R. G. The Aqueous Phase Behavior of Surfactants; Academic Press: New York, 1994. (c) Jönsson, B.; Lindman, B.; Holmberg, K.; Kronberg, B. Surfactants and Polymers in Aqueous Solution; John Wiley \& Sons: West Sussex, 1998. (d) Antonietti, M.; Förster, S. Vesicles and Liposomes: a Self-Assembly Principle beyond Lipids. Adv. Mater. 2003, 15, 1323-1333. (e) Glotzer, S. C.; Solomon, M. J. Anisotropy of Building Blocks and their Assembly into Complex Structures. Nat. Mater. 2007, 6, 557-562. (f) Mai, Y.; Eisenberg, A. Self-Assembly of Block Copolymers. Chem. Soc. Rev. 2012, 41, 5969-5985. (g) Schacher, F. H.; Rupar, P. A.; Manners, I. Functional Block Copolymers: Nanostructured Materials with Emerging Applications. Angew. Chem., Int. Ed. 2012, 51, 78987921. (h) Kang, Y.; Liu, K.; Zhang, X. Supra-Amphiphiles: a New Bridge between Colloidal Science and Supramolecular Chemistry. Langmuir 2014, 30, 5989-6001. (i) Yu, G.; Jie, K.; Huang, F. Supramolecular Amphiphiles Based on Host-Guest Molecular Recognition Motifs. Chem. Rev. 2015, 115, 7240-7303.

(3) (a) Seeman, N. C. Nucleic Acids Junctions and Lattices. J. Theor. Biol. 1982, 99, 237-247. (b) Rothemund, P. W. K. Folding DNA to Create Nanoscale Shapes and Patterns. Nature 2006, 440, 297-302. (c) Aldaye, F. A.; Palmer, A. L.; Sleiman, H. F. Assembling Materials with DNA as the Guide. Science 2008, 321, 1795-1799. (d) Ke, Y.; Ong, L. L.; Shih, W. M.; Yin, P. Three-dimensional Structures SelfAssembled from DNA Bricks. Science 2012, 338, 1177-1183. (e) Hernández-Ainsa, S.; Keyser, U. F. DNA Origami Nanopores: Developments, Challenges and Perspectives. Nanomedicine 2013, 8, $1551-1554$.

(4) (a) Kim, J.; Jung, I.-S.; Kim, S.-Y.; Lee, E.; Kang, J.-K.; Sakamoto, S.; Yamaguchi, K.; Kim, K. New Cucurbituril Homologues: Syntheses, Isolation, Characterization, and X-ray Crystal Structures of Cucurbit$[n]$ uril $(n=5,7$, and 8). J. Am. Chem. Soc. 2000, 122, 540-541. (b) Lagona, J.; Mukhopadhyay, P.; Chakrabarti, S.; Isaacs, L. The Cucurbit[n] uril Family. Angew. Chem., Int. Ed. 2005, 44, 4844-4870. (c) Kim, K.; Selvapalam, N.; Ko, Y. H.; Park, K. M.; Kim, D.; Kim, J. Functionalized Cucurbiturils and their Applications. Chem. Soc. Rev. 2007, 36, 267-279. (d) Dsouza, R. N.; Pischel, U.; Nau, W. M. Fluorescent Dyes and Their Supramolecular Host-Guest Complexes with Macrocycles in Aqueous Solution. Chem. Rev. 2011, 111, 79417980. (e) Barrow, S. J.; Kasera, S.; Rowland, M. J.; del Barrio, J.; Scherman, O. A. Cucurbituril-Based Molecular Recognition. Chem. Rev. 2015, 115, 12320-12406.

(5) (a) Jiao, D.; Biedermann, F.; Tian, F.; Scherman, O. A. A Systems Approach to Controlling Supramolecular Architecture and Emergent Solution Properties via Host-Guest Complexation in Water. J. Am. Chem. Soc. 2010, 132, 15734-15743. (b) Zou, H.; Liu, J.; Li, Y.; Li, X.; Wang, X. Cucurbit[8] uril-Based Polymers and Polymer Materials. Small 2018, 14, 1802234.

(6) del Barrio, J.; Horton, P. N.; Lairez, D.; Lloyd, G. O.; Toprakcioglu, C.; Scherman, O. A. Photocontrol over Cucurbit[8] uril Complexes: Stoichiometry and Supramolecular Polymers. J. Am. Chem. Soc. 2013, 135, 11760-11763. 
(7) Aqueous 1:1 mixtures of $\mathrm{CB}[8]$ and guest molecule 2 (or 3 ) were incubated for ca. $24 \mathrm{~h}$ at $20^{\circ} \mathrm{C}$ prior to use unless otherwise stated.

(8) (a) An, Q.; Chen, Q.; Zhu, W.; Li, Y.; Tao, C.; Yang, H.; Li, Z.; Wan, L.; Tian, H.; Li, G. A Facile Method for Preparing OneMolecule-Thick Free-Standing Organic Nanosheets with Regular Square Shape. Chem. Commun. 2010, 46, 725-727. (b) Lin, F.; Zhan, T. G.; Zhou, T. Y.; Zhang, K. D.; Li, G. Y.; Wu, J.; Zhao, X. The Construction of Rigid Supramolecular Polymers in Water Through the Self-Assembly of Rod-Like Monomers and Cucurbit[8]uril. Chem. Commun. 2014, 50, 7982-7985. (c) Yu, Y.; Li, J.; Zhang, M.; Cao, L.; Isaacs, L. Hydrophobic Monofunctionalized Cucurbit[7]uril Undergoes Self-Inclusion Complexation and Forms Vesicle-Type Assemblies. Chem. Commun. 2015, 51, 3762-3765. (d) Kim, H. J.; Whang, D. R.; Gierschner, J.; Park, S. Y. Highly Enhanced Fluorescence of Supramolecular Polymers Based on a Cyanostilbene Derivative and Cucurbit [8]uril in Aqueous Solution. Angew. Chem., Int. Ed. 2016, 55, 15915-15919. (e) Tian, J.; Chen, L.; Zhang, D.-W.; Liu, Y.; Li, Z.-T. Supramolecular Organic Frameworks: Engineering Periodicity in Water through Host-Guest Chemistry. Chem. Commun. 2016, 52, 6351-6362. (f) Zhang, X.; Wang, C. Supramolecular Amphiphiles. Chem. Soc. Rev. 2011, 40, 94-101. (g) Yan, X.; Zhu, P.; Li. SelfAssembly and Application of Diphenylalanine-Based Nanostructures. Chem. Soc. Rev. 2010, 39, 1877-1890.

(9) The fact that relatively high ionic strength did not hinder aggregation (Figure S11) suggests that hydrophobic interactions contribute to some extent to the formation of higher-order structures.

(10) (a) Lü, J.; Lin, J.-X.; Cao, M.-N.; Cao, R. Cucurbituril: A Promising Organic Building Block for the Design of Coordination Compounds and Beyond. Coord. Chem. Rev. 2013, 257, 1334-1356. (b) Danylyuk, O. Exploring cucurbit[6]uril-peptide interactions in the solid state: crystal structure of cucurbit[6]uril complexes with glycyl-containing dipeptides. CrystEngComm 2017, 19, 3892-3897.

(11) (a) Smith, B. J.; Overholts, A. C.; Hwang, N.; Dichtel, W. R. Insight into the Crystallization of Amorphous Imine-Linked Polymer Networks to 2D Covalent Organic Frameworks. Chem. Commun. 2016, 52, 3690-3693. (b) Tsarfati, Y.; Rosenne, S.; Weissman, H.; Shimon, L. J. W.; Gur, D.; Palmer, B. A.; Rybtchinski, B. Crystallization of Organic Molecules: Nonclassical Mechanism Revealed by Direct Imaging. ACS Cent. Sci. 2018, 4, 1031-1036.

(12) (a) Larson, R. G. The Structure and Rheology of Complex Fluids; Oxford University Press: New York, 1999. (b) Tschoegl, N. S. The phenomenological theory of linear viscoelastic behavior. An introduction; Springer-Verlag: Berlin, 1989.

(13) Cates, M. E. Reptation of Living Polymers: Dynamics of Entangled Polymers in the Presence of Reversible Chain-Scission Reaction. Macromolecules 1987, 20, 2289-2296.

(14) (a) Onsager, L. The Effects of Shape on the Interaction of Colloidal Particles. Ann. N. Y. Acad. Sci. 1949, 51, 627-659. (b) Lyotropic Colloidal and Macromolecular Liquid-Crystals Lekkerkerker, H. N. W.; Vroege, G. J. Lyotropic colloidal and macromolecular liquid crystals. Philos. Trans. R. Soc. London, Ser. A 1993, 344, 419-440.

(15) (a) Avrami, M. Kinetics of Phase Change. I General Theory. J. Chem. Phys. 1939, 7, 1103-1112. (b) Liu, X. Y.; Sawant, P. D. Formation Kinetics of Fractal Nanofiber Networks in Organogels. Appl. Phys. Lett. 2001, 79, 3518-3520.

(16) Henry, M. Nanocrystals from Solutions and Gels. In Encyclopedia of Nanoscience and Nanotechnology; Nalwa, H. S., Ed.; American Scientific Publishers: Valencia, CA, 2004; Vol. 6, p 555.

(17) Supramolecular systems which have been evaluated as drag reducers or viscosity modifiers can be found here: (a) Sabadini, E.; Francisco, K. R.; Bouteiller, L. Bis-Urea-Based Supramolecular Polymer: The First Self-Assembled Drag Reducer for Hydrocarbon Solvents. Langmuir 2010, 26, 1482-1486. (b) Li, L.; Guo, X.; Fu, L.; Prud'homme, R. K.; Lincoln, S. F. Complexation Behavior of $\alpha$-, $\beta$-, and $\gamma$-Cyclodextrin in Modulating and Constructing Polymer Networks. Langmuir 2008, 24, 8290-8296. 